\title{
Implementation of engineering codes for WWER-440 core calculations
}

\author{
Branislav Vrban, Štefan Čerba, Jakub Lüley, Vladimír Nečas
}

\begin{abstract}
The paper gives brief information about the status of the ANDREA code implementation at the Nuclear Regulatory Authority of Slovak republic and Slovak University of Technology in Bratislava. The first results of real fuel loading are compared to KASKAD results and a brief discussion on achieved progress and further plans is provided.

K e y w or d s: ANDREA, KASKAD, comparison, core calculations, implementation phase
\end{abstract}

\section{Introduction}

The primary interest in reactor analysis is to be able to predict and model day-to-day steady state operation of the reactor core, or to model brief periods of time during which the reactor is experiencing some sort of operational transient due to an unexpected insertion or removal of reactivity. Such analyses are performed using a threedimensional nodal code with thermalhydraulic feedback.

Respecting the "safety first" approach, the role of NPP operator (license holder) is to demonstrate that the neutron-physical parameters of the reactor core are in compliance with the design requirements. On the other hand, The Nuclear Regulatory Authority (NRA) should be able to adequately and independently verify the calculation process and results related to such analysis. Following efforts of continuous improvements and strengthening the competency of NRA SR, which exercises state supervision over nuclear safety, the new calculation code ANDREA was procured through a public procurement in late 2016. Since then the implementation phase of the new computational tool is ongoing at NRA SR in a close cooperation with the Institute of Nuclear and Physical Engineering of the Slovak University of Technology in Bratislava (STU).

This paper presents the results of the first implementation phase of the STU computational subgroup and their comparison to characteristics delivered by the license holder to NRA SR. It should be noted, that the intention of this paper is to show the progress of implementation, however some discrepancies may be still observed, due to different computation methods implemented in both codes, historical definitions of calculations paths and not negligible user effect.

\section{The macro code ANDREA}

\subsection{A brief code description}

The macro code ANDREA [1] is an in-house code originally being developed in Nuclear Research Institute (NRI) REZ since 2005. The code is designed to support fuel reload and safety analyses of WWER type reactors. The code solves the two-group diffusion equation with help of contemporary nodal method, which ensures accuracy typical for nodal codes and acceptable speed of calculations. To speed-up the calculations, the conformal mapping of hexagonal homogeneous node onto a rectangular node is implemented resulting in the transfer of 3D diffusion equation into four $1 \mathrm{D}$ diffusion equations related by the transverse neutron leakage terms. The reconstruction of the actual fuel pin-wise power distributions is based on calculated nodal flux and pre-calculated flux shapes for given burnup. The advantage against other codes is the ability to calculate inhomogeneous burnup (burnup gradient) of fuel assemblies. The thermal-hydraulic module assumes the isolated channel for each core subassembly, where the flow-rate is considered to be even in the channels. According to code authors, this assumption is suitable for WWER-440 cores. The program ANDREA carries out the calculation according to the user-defined paths, where operational time, thermal power, control rods position and boric acid concentration can be defined or calculated based on other defined parameters and the actual core status. The program ANDREA has been designed for UNIX-like systems.

\subsection{The cross section libraries}

The design dependent macroscopic cross sections and other constants are comprised in libraries computed in advance by the HELIOS lattice code. These libraries can be prepared by the QUADRIGA user interface; however,

\footnotetext{
*Institute of Nuclear and Physical Engineering, Slovak University of Technology in Bratislava, 81219 Ilkovičova 3, Bratislava, Slovakia, branislav.vrban@stuba.sk
} 


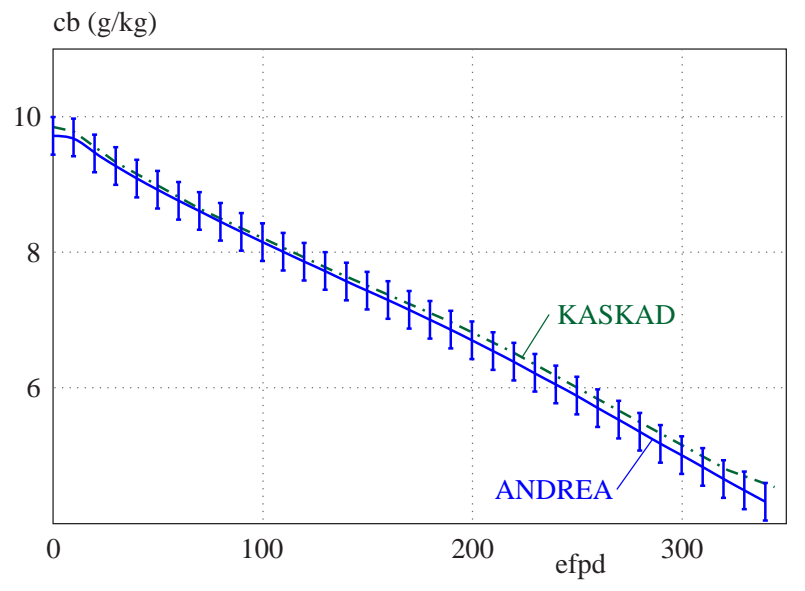

Fig. 1. The critical borid acid concetration for shutdowned core

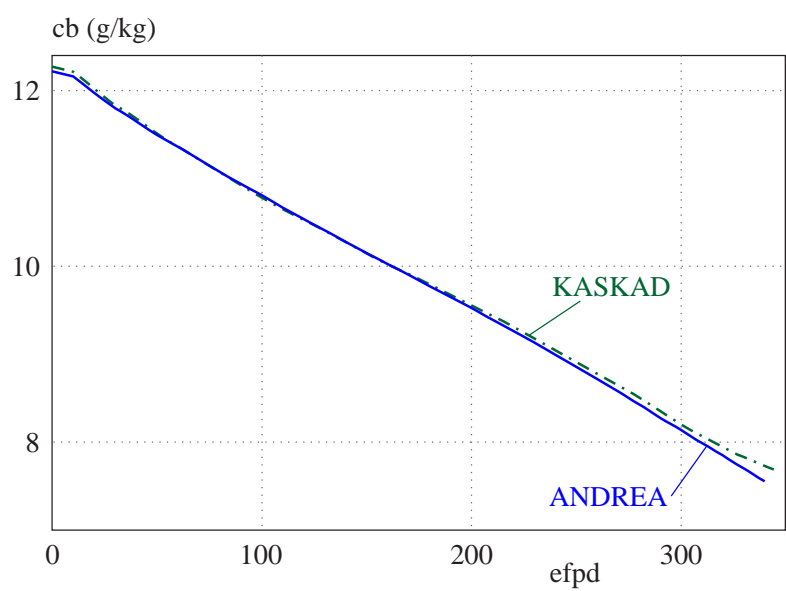

Fig. 2. The borid acid concetration for cold shutdown with SSM and $\rho=-2 \%$

this ability is not included in the license negotiated between NRA SR and NRI REZ. Therefore, the results presented in the paper are based on libraries delivered by NRI REZ to NRA SR and subsequently to STU BA.

\section{The KASKAD package}

The software package KASKAD [2], developed in RRC Kurchatov Institute and intended for calculating the neutronic parameters of WWER reactor cores, is currently used at joint-stock company Slovenské elektrárne, which acts as an operator of Slovak NPPs. The package consists of several coupled codes such as BIPR-7A and PERMAK-A. The BIPR-7A code has been designed to perform 3-D assembly-wise core calculations using the two-group diffusion approximation with $3 \mathrm{D}$ coarse mesh. The PERMAK-A code has been designed to perform pinby-pin multilayer $2 \mathrm{D}$ calculation with 4 or 6 group approximations. Neutronic constant libraries necessary for above mentioned codes are produced by the $2 \mathrm{D}$ spectral code TVS-M [3], while the boundary conditions for radial and axial reflectors can be determined by the PERMAK-A and the PERMAK-3D [4] codes. The whole package is implemented in an interactive graphical inter- face. More information can be found in enclosed references.

\section{Investigated computational paths}

The computational paths for which results are compared here in the paper are chosen based on NRA SRs internal document "The minimum required range of neutron-physical characteristics calculations to control the fuel loadings in Slovak Republic" (hereinafter denoted as Document) [5]. The document is constantly evolving and is periodically changed to reflect the actual findings resulting from implementation activities. All the presented calculations are based on the $33^{\text {rd }}$ fuel loading of NPP Bohunice unit 4.

\section{The actual content of the Document}

The document content is divided to the several sections in which the computational paths are precisely specified. To give a brief overview of the computational volume, the main titles of relevant sections from the Document are shown below.

- The critical boric acid concentrations of different core states including different burnup steps.

- The total control rod (CR) groups worth at the BOC, MOC and EOC conditions.

- Integral and differential characteristics of control rod groups in independent and tandem operation under conditions defined in the previous bullet.

- Additional safety-related parameters of the core, such as axial and radial power peaking factors (nuclear hot channel factors), hot channel position, average core burnup and reactivity feedback coefficients (Doppler feedback, moderator temperature and power feedback).

- The level of subcriticality in case of the most effective CR stuck during shutdown conditions.

- The worth of the most effective CR during withdrawal accident and shutdown conditions.

- The xenon and samarium poisoning effects.

- The kinetic parameters, such as the effective delay neutron fraction and the average prompt neutron lifetime.

- The length of fuel cycle (includes boron regulation, control rod position movement, power and temperature effect).

\section{Methodology of comparison}

Due to the development stage of ANDREA and its implementation phase at NRA SR, the provided comparison is mainly focused on the verification of its usability and abilities. Therefore, the results provided by the license holder (KASKAD) are considered as reference values. Thus, the actual change and the relative change in the investigated parameters are evaluated by (1) and (2). 
Table 1. CR total WORTH-BOC

\begin{tabular}{|c|c|c|c|c|c|c|c|c|}
\hline $\mathrm{N}\left(\% \mathrm{~N}_{\mathrm{nom}}\right)$ & \multicolumn{2}{|c|}{0} & \multirow{3}{*}{\multicolumn{2}{|c|}{$\begin{array}{l}\text { Comparison } \\
\text { to } \\
K A S K A D\end{array}$}} & \multirow{3}{*}{\multicolumn{2}{|c|}{$\begin{array}{c}100 \\
\text { critical } \\
H_{1-6}=250 \mathrm{~cm} \\
T_{m}=268\end{array}$}} & \multirow{3}{*}{\multicolumn{2}{|c|}{$\begin{array}{l}\text { Comparison } \\
\text { to } \\
K A S K A D\end{array}$}} \\
\hline$c b(\mathrm{~g} / \mathrm{kg})$ & \multicolumn{2}{|c|}{13} & & & & & & \\
\hline$\overline{T_{m}\left({ }^{\circ} \mathrm{C}\right)}$ & \multicolumn{2}{|c|}{$T_{m}=27$} & & & & & & \\
\hline- & $\rho(\%)$ & $\mathrm{d} \rho(\%)$ & $\delta \rho(\%)$ & $\delta \mathrm{d} \rho(\%)$ & $\rho(\%)$ & $\mathrm{d} \rho(\%)$ & $\delta \rho(\%)$ & $\delta \mathrm{d} \rho(\%)$ \\
\hline- & -11.18 & 0.00 & -6.5 & 0.0 & -9.62 & 0.00 & -20.5 & 0.0 \\
\hline 1 & -10.44 & -0.74 & -5.3 & -21.3 & -8.80 & -0.82 & -21.4 & -8.9 \\
\hline 2 & -8.76 & -1.67 & -9.0 & 20.1 & -6.38 & -2.43 & -23.5 & -15.0 \\
\hline 3 & -6.56 & -2.20 & -7.2 & -14.1 & -4.18 & -2.20 & -26.0 & -18.2 \\
\hline 4 & -4.85 & -1.72 & -5.5 & -11.3 & -2.29 & -1.89 & -32.4 & -16.4 \\
\hline 5 & -4.58 & -0.27 & -2.3 & -38.6 & -1.62 & -0.67 & -26.4 & -43.7 \\
\hline 6 & -3.46 & -1.12 & 2.7 & -15.2 & -0.00 & -1.62 & 0.0 & -26.4 \\
\hline
\end{tabular}

Table 2. CR total WORTH-EOC

\begin{tabular}{|c|c|c|c|c|c|c|c|c|}
\hline $\mathrm{N}\left(\% \mathrm{~N}_{\text {nom }}\right)$ & \multicolumn{2}{|c|}{0} & \multirow{3}{*}{\multicolumn{2}{|c|}{$\begin{array}{l}\text { Comparison } \\
\text { to } \\
K A S K A D\end{array}$}} & \multirow{3}{*}{\multicolumn{2}{|c|}{$\begin{array}{c}100 \\
\text { critical } \\
H_{1-6}=250 \mathrm{~cm} \\
T_{m}=268\end{array}$}} & \multirow{3}{*}{\multicolumn{2}{|c|}{$\begin{array}{l}\text { Comparison } \\
\text { to } \\
K A S K A D\end{array}$}} \\
\hline$c b(\mathrm{~g} / \mathrm{kg})$ & \multicolumn{2}{|c|}{13} & & & & & & \\
\hline$\overline{T_{m}\left({ }^{\circ} \mathrm{C}\right)}$ & \multicolumn{2}{|c|}{$T_{m}=27$} & & & & & & \\
\hline- & $\rho(\%)$ & $\mathrm{d} \rho(\%)$ & $\delta \rho(\%)$ & $\delta \mathrm{d} \rho(\%)$ & $\rho(\%)$ & $\mathrm{d} \rho(\%)$ & $\delta \rho(\%)$ & $\delta \mathrm{d} \rho(\%)$ \\
\hline- & -18.38 & 0.00 & -3.6 & 0.0 & -9.78 & -9.78 & -19.4 & 0.0 \\
\hline 1 & -17.15 & -1.23 & -3.2 & -8.2 & -8.51 & -8.51 & -22.5 & 10.4 \\
\hline 2 & -15.74 & -1.41 & -6.4 & 54.9 & -6.25 & -6.25 & -24.1 & -17.8 \\
\hline 3 & -13.42 & -2.31 & -6.7 & -4.9 & -4.04 & -4.04 & -27.7 & -16.3 \\
\hline 4 & -11.63 & -1.79 & -4.7 & -17.9 & -2.14 & -2.14 & -35.9 & -15.6 \\
\hline 5 & -11.41 & -0.22 & 0.4 & -73.5 & -1.51 & -1.51 & -28.1 & -49.2 \\
\hline 6 & -10.43 & -0.98 & 2.5 & -17.6 & -0.00 & -0.00 & 0.0 & -28.1 \\
\hline
\end{tabular}

The relative change can be expressed in percentage, if necessary.

$$
\begin{gathered}
\Delta=A N D R E A-K A S K A D, \\
\delta=\Delta / K A S K A D .
\end{gathered}
$$

\section{Ressults}

For the sake of brevity, just exemplary results are provided in the paper. The results of boric acid concentrations $(c b)$ for different effective power days $(e f p d)$, shutdown condition $(P=0)$, the average moderator/coolant temperature $T_{m}=200^{\circ} \mathrm{C}$, the position of the 6 -th $\mathrm{CR}$ group $H_{6}=175 \mathrm{~cm}$, the 6 -th $\mathrm{CR}$ group position through nominal power operation $(\mathrm{NPO}) H_{6 \mathrm{NPO}}=225 \mathrm{~cm}$ and no xenon poisoning $(X e=0)$ are shown in Fig. 1 . The value calculated by the ANDREA code is bounded by symmetric uncertainty at $95 \%$ confidence level $(0.3 \mathrm{~g} / \mathrm{kg})$ as defined in the document [6]. The relative change is always below $3 \%$ and the KASKAD values lies inside the
ANDREA uncertainty. These calculations may be considered as identical.

The second category of the presented results is the concentration of boric acid during cold shutdown with CRs parked in their upper-end positions and with reactivity level of $-2 \%$. As defined, the additional safety shutdown margin $(\mathrm{SSM}=0.3 \mathrm{~g} / \mathrm{kg})$ is applied to the calculated concentrations. Fig. 2 shows the comparison of results achieved by both codes. As it is clear from Fig. 2, the maximal relative change of final concentrations is under $1 \%$, therefore these calculations can be considered as identical.

The CRs (37 pcs.) are in WWER-440 divided to 6 groups, where the sixth group consists of 7 pcs. including the central rod and the other groups consist of 6 pcs. The worth of each specific group is calculated by its subsequent withdrawal to the upper parking position from the initial core state, where all control rods were fully inserted to the core. The reactivity worth of a specific group is then calculated as the difference between the reactivity of the inserted CRs and the reactivity of the CRs in their upper parking positions. Several core states (power level, coolant temperature, burnup level) 
Table 3. The radial power peaking factors

\begin{tabular}{lccccccc}
\hline Parameter & BOC & MOC & EOC & Parameter & BOC & MOC & EOC \\
\hline FR & 1.353 & 1.392 & 1.362 & FL & 1.858 & 1.801 & 1.726 \\
FA position & 35 & 35 & 35 & FA position & 26 & 26 & 26 \\
$\delta$ FR & $0.97 \%$ & $1.27 \%$ & $0.58 \%$ & $\delta \mathrm{FL}$ & $1.75 \%$ & $1.07 \%$ & $3.60 \%$ \\
\hline
\end{tabular}

Table 4. The reactivity coefficients

\begin{tabular}{lcccc}
\hline $\begin{array}{l}\text { Parameter } \\
\text { Unit }\end{array}$ & $\begin{array}{c}\text { Moderator } \mathrm{RC} \delta \rho / \delta \mathrm{T}_{\mathrm{M}} \\
\left(10^{-2} \% /{ }^{\circ} \mathrm{C}\right)\end{array}$ & $\begin{array}{c}\text { Total } \mathrm{RC} \delta \rho / \delta \mathrm{T} \\
\left(10^{-2} \% /{ }^{\circ} \mathrm{C}\right)\end{array}$ & $\begin{array}{c}\text { Moderator } \mathrm{RC} \delta \rho / \delta \mathrm{T}_{\mathrm{M}} \\
\left(10^{-2} \% /{ }^{\circ} \mathrm{C}\right)\end{array}$ & $\begin{array}{c}\text { Total } \mathrm{RC} \delta \rho / \delta \mathrm{T} \\
\left(10^{-2} \% /{ }^{\circ} \mathrm{C}\right)\end{array}$ \\
\hline BOC & -0.119 & -0.467 & 0.028 & -0.323 \\
$\Delta$ & -0.056 & -0.085 & -0.054 & -0.084 \\
MOC & -0.519 & -0.875 & -0.368 & -0.727 \\
$\Delta$ & -0.041 & -0.066 & -0.039 & -0.065 \\
EOC & -1.142 & -1.506 & -0.988 & -1.356 \\
$\Delta$ & -0.121 & -0.146 & -0.120 & -0.146 \\
\hline
\end{tabular}

Table 5. The CR worth during withdrawal accident

\begin{tabular}{lccc}
\hline Parameter & BOC & MOC & EOC \\
\hline $\mathrm{d} \rho$ & 0.62 & 0.57 & 0.56 \\
$\delta$ & $5.08 \%$ & $0.00 \%$ & $-1.75 \%$ \\
\hline
\end{tabular}

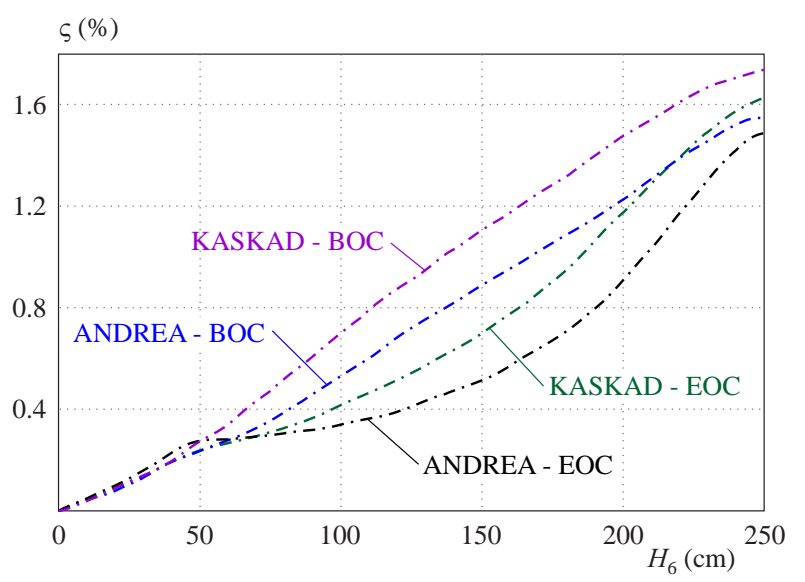

Fig. 3. The $\mathrm{S}$ curves of the $6^{\text {th }} \mathrm{CR}$ group with the $5^{\text {th }}$ group in tandem

were investigated, but just only four specific cases are presented here. The first case comprises the absolute results $(\rho \& \mathrm{~d} \rho)$ and relative change values $(\delta \rho \& \delta \mathrm{d} \rho)$ for BOC - cold shutdown with boron acid concentration equal to $c b=13 \mathrm{~g} / \mathrm{kg}$. The second case is defined as BOC - full power, critical boron acid concentration and the 6 -th $\mathrm{CR}$ group position $H_{1-6}=250 \mathrm{~cm}$. Results can be found in Tab. 1.

The symmetric uncertainty of ANDREA regarding the effectiveness of CR groups is $20 \%$. The relative change of the cold shutdown state shown in Tab. 1 are well below or close to requested margin, however data which belong to the full power case are much more biased. Here the maximal relative change reaches approximately $44 \%$. It should be noted that the actual changes are not so dramatic, nevertheless the source of these discrepancies need to be clarified in the future. The structurally same results at the EOC can be found in Tab. 2 .

From Tab. 2 a reader can recognize that relative changes are relatively high for the cold-shutdown state. The results for the full power are almost identical with BOC condition. It can be considered that these discrepancies from both codes are systematically biased, but authors assume that the results are mainly influenced by the different calculation scheme applied by different teams (user effect). These inconsistencies can be clearly seen also on the CR S curves. As an example, the S curves of the $6^{\text {th }} \mathrm{CR}$ group with the $5^{\text {th }}$ group in tandem are shown in Fig. 3 at BOC and EOC conditions. The average moderator/coolant temperature is set up to $T_{m}=200^{\circ} \mathrm{C}$ and the core is in shutdown state. If the total worth of each CR group presented in Fig. 3 is normalized to unity, it can be realized that the reactivity shift of the $\mathrm{CR}$ worth from BOC to EOC conditions is preserved for both codes. This fact can help to find the reason of biased results.

In general, power peaking factors are important for steady state core operation. The results of the radial power peaking factors (FRs) and the linear power peaking factors (FLs) are presented in this section. The FR is defined as the ratio between the maximum generated power of actual fuel assembly (FA) and the average power of one FA in the core. The linear power peaking factor is defined as the linear power rate of actual position to the average level of linear power rate in the core. The maximal FRs and FLs and corresponding positions of FAs were compared to KASKAD results. The results of ANDREA code and relative changes at $\mathrm{BOC}, \mathrm{MOC}$ and EOC conditions, $H_{6 \mathrm{NPO}}=226.2 \mathrm{~cm}$ and $H_{6}=226.2 \mathrm{~cm}$ are shown in Tab. 3. 
Table 6. Sub-criticality of the core in case of stuck CRr of the $6^{\text {th }}$ group

\begin{tabular}{lcccccc}
\hline \multirow{2}{*}{$\mathrm{T}_{\mathrm{M}}\left({ }^{\circ} \mathrm{C}\right)$} & \multicolumn{2}{c}{ BOC } & \multicolumn{2}{c}{ MOC } & \multicolumn{2}{c}{ EOC } \\
& $\rho(\%)$ & $\Delta$ & $\rho(\%)$ & $\Delta$ & $\rho(\%)$ & $\Delta$ \\
\hline 260 & -2.939 & 2.093 & -2.983 & 1.795 & -2.501 & 2.135 \\
240 & -2.220 & 1.967 & -2.096 & 1.695 & -1.385 & 2.080 \\
200 & -1.127 & 1.733 & -0.724 & 1.508 & 0.380 & 1.982 \\
153 & -0.188 & 1.460 & 0.480 & 1.262 & 2.207 & 2.207 \\
120 & 0.284 & 1.332 & 1.099 & 1.177 & - & - \\
117 & 0.380 & 1.308 & 1.214 & 1.214 & - & - \\
80 & 0.756 & 1.118 & - & - & - & - \\
52 & 1.018 & 1.018 & - & - & - & - \\
\hline
\end{tabular}

Table 7. The kinetic parameters

\begin{tabular}{lcc}
\hline Parameter & $\beta_{\text {eff }} \times 10^{-2}$ & $\Lambda_{\text {str }} \times 10^{-4}(\mathrm{~s})$ \\
\hline BOC & 0.6 & 0.19 \\
$\delta$ & $-3.23 \%$ & $11.76 \%$ \\
EOC & 0.55 & 0.21 \\
$\delta$ & $-3.51 \%$ & $5.00 \%$ \\
\hline
\end{tabular}

As can be seen in Tab. 3, the relative change for both coefficients is less than $4 \%$ in all cases, what can be considered as acceptable. Also, the corresponding FA positions are correctly identified. However, the different definitions of the ratio of the total pin power to the total core power divided by number of pins $(\mathrm{FdH})$ are used in both computational systems. This discrepancy can be solved by the correct definition of desired computational path.

A reactivity coefficient $(\mathrm{RC})$ is defined as the change of reactivity per unit change in some operating parameter of the reactor. The moderator temperature coefficient (MJC) and total reactivity coefficient (calculated as a sum of MJC and FTC - fuel temperature coefficient) were investigated due to their importance for evaluation of the limits and condition of safe operation within each reactor campaign. ANDREA systematically underestimates the moderator temperature coefficient by $0.25 \times 10^{-2} \% /{ }^{\circ} \mathrm{C}$, which was statistically evaluated within ANDREA code validation. If the bias is applied, the standard deviation of $0.25 \times 10^{-2} \% /{ }^{\circ} \mathrm{C}$ can be considered for the final value of moderator temperature coefficient. The results calculated by ANDREA for critical and sub-critical reactor state and the associated absolute changes can be found in Tab. 4.

The actual changes of Moderator and Total RC are in all cases within the standard deviation of $0.25 \times$ $10^{-2} \% /{ }^{\circ} \mathrm{C}$ where the same conditions as for Moderator $\mathrm{RC}$ as well as for Total RC were considered due to usage of the biased Moderator RC within determination of the Total RC and no information about Total RC uncertainties exist. Generally, almost perfect agreement was obtained in comparison of the codes ANDREA and KASKAD. For the BOC the absolute change is relatively small, but to- wards to higher depletion is increasing. This increase insignificant compare to increase of the values of RCs and therefore can be also consider as negligible.

The results of the worth of the most effective CR during withdrawal accident and shutdown conditions are presented in Table 5. Zero power was assumed before the $\mathrm{CR}$ withdrawal $(P=0), T_{m}=260^{\circ} \mathrm{C}, H_{6 \mathrm{NPO}}=$ $226.2 \mathrm{~cm}$ and the position of the $6^{\text {th }} \mathrm{CR}$ group was $H_{6}=$ $50 \mathrm{~cm}$. According to this calculation, the most effective $\mathrm{CR}$ is the one placed on the core periphery in position 6.6. The results demonstrated in Tab. 5 are in good agreement where the maximal relative change of $\mathrm{CR}$ worth equals approximately $5 \%$. Even more, the calculated results are same at MOC. These results can be judged as consistent and acceptable.

The comparison of results for the sub-criticality of the core in case of stuck CR of the $6^{\text {th }}$ group between ANDREA and KASKAD is shown in Tab. 6. In the calculation the critical concentration of boric acid was calculated for the position of the $6^{\text {th }} \mathrm{CR}$ group $H_{6 \mathrm{NPO}}=226.2 \mathrm{~cm}$. Subsequently the position of the sixth CR group was changed to $H_{6}=172.6 \mathrm{~cm}$, the power of the core was set to zero power state and the sub-criticality of the core was calculated for the set of temperatures defined in Tab. 6 . From the results we can see large discrepancy between the ANDREA and the KASKAD results, which exceeds the statistical uncertainty of the calculation of control rod worth in ANDREA $(20 \%)$. The source of this discrepancy will have to be analysed in the future.

The next results are devoted to the kinetic parameters important for the reactor control system and related reactimeters. The effective delayed neutron fraction $\left(\beta_{\text {eff }}\right)$ values and the average neutron generation times $\left(\Lambda_{\mathrm{avg}}\right)$ calculated by ANDREA at BOC and EOC conditions are presented in Tab. 7 .

The results in the table above show good agreement of the delayed neutron fractions where the relative change is lower than $4 \%$. The results of the average neutron times are slightly worse, where the relative change at BOC reaches almost $12 \%$. It is hard to predict, whether these discrepancies come from nuclear data or they are caused by the different isotopic composition at the BOC. 
Table 8. The calculated lengths of fuel cycle

\begin{tabular}{lcccc}
\hline $\begin{array}{c}H_{6} \\
\mathrm{~cm}\end{array}$ & $\begin{array}{c}N \\
\text { \%nom }\end{array}$ & $\begin{array}{c}T_{\mathrm{m}} \\
{ }^{\circ} \mathrm{C}\end{array}$ & $\begin{array}{c}\text { average fuel burnup } \\
\mathrm{MWd} / \mathrm{kgU}\end{array}$ & $\begin{array}{c}\text { Length of fuel cycle } \\
\text { efpd }\end{array}$ \\
\hline 226.0 & 100 & 284.8 & 36.97 & 316.73 \\
& $\delta$ & $0.80 \%$ & $-0.22 \%$ & $-0.89 \%$ \\
250.1 & \multirow{2}{*}{100} & 284.4 & 37.17 & 322.84 \\
& $\delta$ & $0.66 \%$ & $-0.24 \%$ & $-0.95 \%$ \\
250.1 & 97.84 & 284 & 37.25 & 325.22 \\
& $\delta$ & $1.39 \%$ & $-0.75 \%$ & $-2.63 \%$ \\
\hline
\end{tabular}

The comparison of kinetic parameters should be made for the first imaginary fuel loadings in future.

The calculated lengths of fuel cycle for boron regulation, for the different positions of the $6^{\text {th }}$ CR group and power levels are shown in Tab. 8. The comparisons quantified using the relative changes show a very good agreement and consistency between both codes. It can be seen that the relative deviations are well below $1 \%$ for nominal power cases. The length of fuel cycle measured in effective power days differs more for the case of decreased power, however the relative deviation below $3 \%$ is still acceptable and lies within the uncertainty declared by ANDREA developers. The relative changes of average moderator/coolant temperatures Tm are below $2 \%$ and can be judged as negligible.

\section{Conclusion}

The abilities of ANDREA code were demonstrated by comparing its results with the KASKAD code package. Taking into account the implementation phase of the ANDREA code at NRA SR and STU in Bratislava, the achieved results can be considered as satisfactory. Nevertheless, the discrepancies in the worth of CR groups need to be further investigated and clarified. The reliable option of further work is the Monte Carlo calculation of the CR worth for zero power reactor. The same applies to the power reactivity feedback where the relative changes exceed the level of $100 \%$. Based on results, authors assume that the most significant differences between codes are reflector macroscopic cross section data and simplifications used in the thermal hydraulic model. Authors believe that successful implementation of the ANDREA code will extent the abilities of NRA SR and therefore will improve the nuclear safety in Slovakia.

\section{Acknowledgement}

Authors would like to thank MSc Michal Melichrek, inspector of nuclear safety of NRA SR for organising and lead of implementation activities of code ANDREA at NRA SR. This study has been partially financially supported by the Slovak Research Development Agency No. APVV-16-0288 and the Slovak Academy of Sciences No. VEGA 1/0863/17.

\section{REFERENCES}

[1] F. Havlůj, R. Vočka and J. Vysoudil, "ANDREA 2: Improved Version of Code for Reactor Core Analysis", Procedings of the $22^{\text {nd }}$ International conference on Nuclear Engineering, Prague, Czech Republic, July 7-11, 2014.

[2] P. A. Bolobov, A. P. Lazarenko and M. Ju. Tomilov, "Development of the Code Package KASKAD for Calculations of WWERs", Proceedings of the eighteenth symposium of atomic energy research, Hungary, 2008.

[3] V. D. Sidorenko et al, "Spectral Code TVS-M for Calculation of Characteristics of Cells, Supercells and Fuel Assemblies of VVER-type Reactors", Proceedings of $5^{\text {th }}$ Symposium of the AER, Dobogóko, Hungary, Oct 15-20, 1995.

[4] A. I. Scherenko, P. A. Bolobov, A. P. Lazarenko and M. Ju, "Using of the Code Package KASKAD for Calculations of VVER Cores Neutronic Parameters", IAEA Regional training course \#3 on infrastructure development and safety assesment of WWER/PWR with advanced safety features, Obninsk, Russian Federation, July 17-28, 2017, unpublished.

[5] M. Melichárek, V. Adamovský, M. Antal, Š. Čerba, J. Lúley and B. Vrban, "The Minimum Required Range of Neutron-Physical Characteristics Calculations to Control the Fuel Loadings in Slovak Republic V3", NRA SR, 2017, unpublished.

[6] J. Hejzlar and R. Vočka, "Qualification of the ANDREA Computing Program, Version 2.2", 2016, unpublished.

Received 1 November 2018 